\title{
Subject Index Vol. 36, 1996
}

Accelerometry 29

Acoustic analysis 310

Acute disseminated encephalomyelitis 219

- stroke 13

Acyclovir 288

Aging 138

Agonist and antagonist electromyographic

activity 333 AIDS 71,229 Albumin ratio 201 Alien hand syndrome 39 Alkaline phosphatase 300 Alzheimer's disease 103, 149, 197, 378 Anticholinergic drugs 164 Antineuronal nuclear antibodies 369 Aphasia activity 333 AII

Arnold-Chiari malformation 36 Assessment 48

Asymptomatic cerebral infarction 343 Ataxic dysarthria 310 Autoantibodies 138 Autonomic cardiovascular system 211 Axons 3

Basal ganglia 293 Behaviour disturbances 146 Blood-cerebrospinal fluid barrier 201 Bone formation 300

- resorption 300

Botulinum toxin A 29

Bromocriptine therapy 164

Carbon disulfide polyneuropathy 364 Carotid duplex sonography 364 Case-control study 85 CD4+cells 125 Cephalometry 206 Cerebellar dysmetria 333 Cerebellum 310 Cerebral atrophy 268

- edema 25

- infarction 343

- ischemia 76 Cerebrovascular disease 25 Charcot-Marie-Tooth syndrome 224 Childhood 79

Chlamydia 160 Chromosome 17224 Coenzyme Q io 191 Cognition 125 Computed tomography 364 Convexity meningioma 43

Corona radiata 338 Cortical isolation 374 Corticobasal degeneration 134 Cross-links 300 Cryoglobulinemia 138 CT/scans 353 Cytochrome c oxidase 260

Deficient skill learning 154 Dementia 94, 268, 378 Demyelinating diseases 79

- disorders 219

Denial 353

Depression 293

Diabetes mellitus 303

Diagnosis 260

Differential diagnosis 378

DNA analysis 348

-, mitochondrial 260 Dorsal root ganglia 3

Early-stage Parkinson's disease 164 Electroencephalography 303, 374 Electron transport 260 Electrophysiological modelization 224 Encephalitis 160,219 Encephalopathy 364 Epidemiology 103 Epilepsy 146,273 Epileptic aphasia 374

Felbamate 146

Functional magnetic resonance imaging

117 Fundamental frequency 310

Gangliosides 138 Gottfries-Brane-Steen scale 315

Headache 229

Hemineglect 385

Hemiplegia 278

Hemoglobin 85

Hereditary motor and sensory neuropathy

type 1224 Herpes zoster 288 Hip fracture 278 Hippocampus 94 Histochemistry 98 Human immunodeficiency virus 125

,-- typel 229

- - - infection 71 Huntington's disease 348

Hydroxyproline 300 Hypertensive encephalopathy 25 Hypoglycemia 303

Ideomotor apraxia 39 Immunoglobulin M index 201 Implicit memory 154 Inclusion body myositis 89 Infection 160

Instrumental measurement 310 Intellectual and cognitive function 315 Interhemispheric subdural hematoma 43 Internal borderzone infarction 343

- capsule 338

Intestinal pseudo-obstruction 369 Intracranial occlusive disease 65 Intrathecal synthesis 201 Ischemic stroke 85 Isokinetic muscle strength 284 IT15gene 348

Lacunar stroke 338 Lacune 343 Late-onset epilepsy 20 Leukoaraiosis 20 Lymphoma 138

Magnetic resonance imaging 215, 219, 268, 293,364 
- volumetry 273

Mapping 303

Mental deterioration 20 Middle cerebral artery 65 Migraine 76 Mini Mental State examination 315

- - - test 197 Mitochondria 149,260 Mitochondrial cytopathy 98

- encephalomyopathies 260

- myopathy 191 Monoclonal gammopathy 138 mtDNA deletion 191 Mueller maneuver 206 Multi-infarct dementia 315 Multiple sclerosis 79, 211,374 Muscle enzymes 142 Myoglobinuria 142 Myotonia congenita 284 Myotonic inhibition 284

Neglect 353

Nerve conduction velocities 224

- injury 3

411

Neurodegenerative disorders 149 Neurofibrillary tangle 94 Neurological signs 71 Neuropathy 3

Neuropsychological markers 125 Neuropsychology 353, 378 Neurotoxic disease 364 New anti-epileptic drugs 146 Non-dystonic tremors 29

Organic brain syndrome 303 Osteopenia 278 Oxygen desaturation 206

${ }^{31} \mathrm{P}$ magnetic resonance spectroscopy 191

p24 antigen 125

Pain 229

Paraneoplastic syndrome 369

Paresthesia 3

Parkinson's disease 154,164

Patch clamp 3

Patent foramen ovale 76

Pentoxifylline 315

Periodic lateralized epileptiform discharges

374 Perseveration 39 Phenytoin overdosage 273 Pick's disease 134 Placebo 29

Point mutation 149 Pons 338

Positron emission tomography 20 Postherpetic neuralgia 288 Post-polio syndrome 89 Primary progressive aphasia 134 Procedural learning 154 Prognosis 103

Quail poisoning 142 Quantitative EEG 197

Ragged-red fiber(s) 13,98 Recovery 48 Rehabilitation 385 Respiratory control 206

- failure 13,36

Risk factors 85

Sandoz Clinical Assessment Geriatric scale

315 Scapuloperoneal syndrome 89 Scleroderma 89 Segmental motor paresis 288 Senile plaque 94 Sensory neuronopathy 369

- neuropathy 138

Signal hyperintensity 293

Silent infarction 343

Skeletal muscle 13,284 Sleep 211

- apnea 36,206 Sodium channels 3

- current 3 Somatotopy 338 SPECT 197 Sporadic cases 348 Status epilepticus 374 Stroke 268, 278, 353, 385 Sulfatide 138

Surface electromyography 29 Sympathetic innervation 25 Syringobulbia 215 Syringomyelia 215

Taiwanese 310 Tetrodotoxin 3 Tone language 310

Ultrasound 65

Upper airway obstruction 206

Urinary retention 43

Ventricular enlargement 293

Voice onset time 310

Voluntary motor performance 284

Whiplash injury 48 
